\title{
High-pressure and temperature autoclaving of peanuts reduces the proportion of intact allergenic proteins
}

\author{
Casey Cohen ${ }^{1}$, Wei Zhao ${ }^{1}$, Liane Beaudette ${ }^{2}$, Duncan Lejtenyi ${ }^{2}$, Bertrand Jean-Claude ${ }^{1}$, \\ and Bruce Mazer ${ }^{1}$ \\ ${ }^{1}$ Research Institute of the McGill University Health Centre \\ ${ }^{2}$ Montreal Children's Hospital
}

April 29, 2021

\begin{abstract}
High-pressure and temperature autoclaving of peanuts reduces the proportion of intact allergenic proteins
\end{abstract}

Casey G. Cohen ${ }^{\mathrm{a}}$, Wei Zhao ${ }^{\mathrm{a}}$, Liane Beaudette ${ }^{\mathrm{b}}$, Duncan Lejtenyi ${ }^{\mathrm{b}}$, Bertrand J. Jean-Claude ${ }^{\mathrm{a}}$, Bruce D. Mazer $^{\mathrm{a}, \mathrm{b}}$

To the Editor,

Peanut allergy is extremely common, affecting approximately 1.5\% of children in North America, Australia and the UK ${ }^{1}$. Peanut does not appear to denature under normal cooking conditions. Structural biology analyses have focused on the three-dimensional structure of major peanut protein allergens such as Ara $\mathrm{h} 1$ and Ara h 2. This class of proteins is rich in disulfide bridges, which explains their resistance to denaturation at high temperature ${ }^{2}$. Analysis of whole autoclaved roasted peanuts demonstrated a decrease of IgE-binding capacity of peanut allergens and in wheal size by skin prick test, as well as the unfolding of proteins and reduction in overall secondary structure ${ }^{3}$. The objective of this study was to evaluate the effects of thermal processing, particularly roasting and autoclaving, on the allergenicity of major peanut protein allergens Ara h2 and Ara h8.

Western blot analyses were performed on protein extracts of each processing condition. In all autoclaved extracts, no distinct bands were observed for Ara h 2 or Ara h 8, while high levels of band intensity were observed for the raw and roasted extracts (Figure 1A). For both Ara h 2 and Ara h 8, raw and roasted protein extracts showed high levels of detection by ELISA (Figure 1B). However, for the autoclaved extract, detection was reduced by approximately $50 \%$ for Ara h 2, while no Ara h 8 was detected in the autoclaved extracts, independent of extract concentration (Figure 1B). Furthermore, $\operatorname{IgE}$ binding decreased significantly upon autoclaving peanut (Figure 1C, p $<0.0001$ ), as shown by ELISA using the sera of 4 highly allergic patients (ImmunoCAP > $50 \mathrm{IU}$ ).

Nine peanut-allergic individuals were subjected to skin prick tests (SPT) with a panel of protein extracts derived from raw, roasted and autoclaved peanuts and the resulting wheal diameters were measured (Table I). Within the peanut-allergic group, a statistically significant reduction in mean wheal diameter was observed using the autoclaved extract when compared to raw $(\mathrm{p}<0.01)$. Additional information was revealed when the allergic group was further stratified into two groups: (1) patients who have previously experienced systemic symptoms or anaphylaxis to peanut or have demonstrated high likelihood based on past SPT and serum-IgE tests, and (2) those who have experienced only oral symptoms upon peanut consumption. A striking decrease in wheal size was observed when using autoclaved extracts compared to raw in the group that experiences oral symptoms. In contrast, this was not observed in the group at risk for anaphylaxis. 
Our discovery of complete and partial degradation of Ara h 8 and Ara h 2, respectively, under autoclaving may have significant clinical implications. Currently, whole protein extracts created from raw or roasted peanuts are routinely used in SPTs for the diagnosis of peanut allergy in the clinic. Sensitivity to Ara $h$ 2 has proven to be one of the best predictors of anaphylaxis in allergic patients ${ }^{4}$, while isolated Ara h 8 sensitization correlates with oral symptoms or tolerance to peanut in almost all cases ${ }^{5}$. Our results indicate that the use of an autoclaved peanut extract, in addition to the current whole protein extract, has the potential to serve as an improved diagnostic tool (patent applied ${ }^{6}$ ), distinguishing between two subsets of peanut-allergic patients: (1) those at risk for anaphylaxis, and primarily have Ara h 2 -specific IgE, versus (2) those who will only experience oral symptoms to peanut, and predominantly present Ara $\mathrm{h}$ 8-specific IgE. As depicted in Figure 1D, patients with a positive SPT result using both the whole peanut extract (raw or roasted) and the autoclaved peanut extract will be classified as at risk for anaphylaxis (subset 1). Importantly, patients with a positive SPT result using the whole peanut extract, but a negative SPT result using the autoclaved extract, will experience only oral symptoms upon peanut consumption (subset 2). Patients tolerant to peanut will experience a negative SPT result with both extracts.

Altogether, the data reported in this study suggest that high-pressure and temperature autoclaving leads to a significant denaturation of allergenic peanut proteins. This discovery is being further developed into an improved diagnostic test for peanut-allergic patient stratification. Further studies are required to optimize a degree of complete reduction of intact allergens by autoclaving.

Table I. Skin Prick Test (SPT) results displaying the mean wheal diameter in millimetres.

\begin{tabular}{|c|c|c|c|c|}
\hline \multirow{4}{*}{ Extract used } & Mean Wheal & Mean Wheal & Mean Wheal & Mean Wheal \\
\hline & Diameter (mm) & Diameter (mm) & Diameter (mm) & Diameter (mm) \\
\hline & Allergic $^{+}(\mathrm{n}=9)$ & Allergic $^{+}(\mathrm{n}=9)$ & Allergic $^{+}(\mathrm{n}=9)$ & $\begin{array}{l}\text { Non-Allergic }(\mathrm{n}= \\
3)\end{array}$ \\
\hline & & $\begin{array}{l}\text { Anaphylaxis }(\mathrm{n}= \\
5)\end{array}$ & $\begin{array}{l}\text { Oral Symptoms } \\
(\mathrm{n}=4)\end{array}$ & \\
\hline Standard & 10.2 & 12.8 & 7.0 & 1.3 \\
\hline Raw & 9.6 & 12.4 & 6.0 & 1.0 \\
\hline Roast & 9.9 & 9.0 & 11.0 & 1.3 \\
\hline Autoclave & $6.9 * *$ & 11.0 & $1.8^{*}$ & 1.0 \\
\hline $\begin{array}{l}\text { Autoclave- } \\
\text { Roasted }\end{array}$ & 6.7 & 10.2 & 2.3 & 1.3 \\
\hline $\begin{array}{l}\text { Roasted- } \\
\text { Autoclave }\end{array}$ & 6.9 & 10.6 & 2.3 & 1.0 \\
\hline $\begin{array}{l}(+) \text { Control } \\
\text { (Histamine) }\end{array}$ & 4.2 & 4.2 & 4.3 & 4.3 \\
\hline $\begin{array}{l}\text { (-) Control } \\
\text { (Saline) }\end{array}$ & 0.7 & 0.6 & 0.8 & 1.3 \\
\hline
\end{tabular}

*: $\mathrm{p}<0.05,{ }^{* *}: \mathrm{p}<0.01$, Student's $t$-test compared to raw.

+ Allergic subjects were divided into two sub-groups based on previous exposure to peanut: those at risk for anaphylaxis and those who experience only oral symptoms.

\section{Hosted file}

image1.emf available at https://authorea.com/users/410875/articles/520086-high-pressure-andtemperature-autoclaving-of-peanuts-reduces-the-proportion-of-intact-allergenic-proteins

FIGURE 1. A, Western blot following SDS PAGE using antibodies specific for Ara h 2 and Ara h 8. Auto-Roast: autoclaved, then roasted. Roast-Auto: roasted, then autoclaved. L: Molecular weight ladder. B, Relative Ara h 2 and Ara h 8 quantification by ELISA. Optical density (OD) values were measured at 
$450 \mathrm{~nm}$ and referenced at $570 \mathrm{~nm}$. Plates were coated with a maximum concentration of $1 \mu \mathrm{g} / \mathrm{mL}$ (Ara h 2) and $1 \mathrm{mg} / \mathrm{mL}$ (Ara h 8) peanut protein. C,Peanut-Specific IgE ELISA using the serum of 4 highly allergic subjects. OD values measured at $450 \mathrm{~nm}$, referenced at $570 \mathrm{~nm}$, were normalized to corresponding raw values. $\mathrm{n}=4$ patients. ****: $\mathrm{p}<0.0001$, one-way ANOVA, Tukey's multiple comparisons test. D, Summary of hypothesized effect of autoclaving on peanut allergens Ara h 2 and Ara h 8 when compared to raw or roasted allergens and expected outcomes of proposed diagnostic method. $50 \%$ and $0 \%$ of intact Ara $\mathrm{h}$ 2 and Ara h 8, respectively, can be detected in autoclaved peanut extracts when compared to raw or roasted extracts. Highlighted areas in red represent epitope regions in protein structures. When using the whole protein extract made from raw or roasted peanuts for Skin Prick Testing (SPT) as currently done in the clinic, patients who have IgE specific for any combination of peanut allergens will experience a positive SPT result. However, when using the autoclaved extract, patients at risk for anaphylaxis (specific IgE primarily for Ara h 2) will experience a positive SPT result while patients who experience only oral symptoms to peanut (specific IgE primarily for Ara h 8) will experience a negative SPT result.

References

1. Ben-Shoshan, M.; Turnbull, E.; Clarke, A., Food Allergy: Temporal Trends and Determinants. Curr. Allergy Asthma Rep. 2012,12 (4), 346-372.

2. Koppelman, S. J.; Hefle, S. L.; Taylor, S. L.; de Jong, G. A. H., Digestion of peanut allergens Ara h 1, Ara h 2, Ara h 3, and Ara h 6: A comparative $<\mathrm{i}>$ in vitro $</ \mathrm{i}>$ study and partial characterization of digestion-resistant peptides. Mol. Nutr. Food Res. 2010,54 (12), 1711-1721.

3. Cabanillas, B.; Maleki, S. J.; Rodriguez, J.; Burbano, C.; Muzquiz, M.; Jimenez, M. A.; Pedrosa, M. M.; Cuadrado, C.; Crespo, J. F., Heat and pressure treatments effects on peanut allergenicity. Food Chem. 2012, 132 (1), 360-6.

4. Kukkonen, A. K.; Pelkonen, A. S.; Makinen-Kiljunen, S.; Voutilainen, H.; Makela, M. J., Ara h 2 and Ara 6 are the best predictors of severe peanut allergy: a double-blind placebo-controlled study. Allergy 2015, 70 (10), 1239-1245.

5. Asarnoj, A.; Nilsson, C.; Lidholm, J.; Glaumann, S.; Ostblom, E.; Hedlin, G.; van Hage, M.; Lilja, G.; Wickman, M., Peanut component Ara h 8 sensitization and tolerance to peanut. J. Allergy Clin. Immunol. 2012, 130 (2), 468-72.

6. Cohen, C.; Jean-Claude, B. J.; Mazer, B. Peanut Hypoallergenic Formulations for Determining the Risk of Anaphylaxis. 2020.

KEYWORDS: Autoclaving, Food Processing, IgE Binding, Peanut Allergy, Skin Prick Testing

CONFLICT OF INTEREST: The authors have no conflicts of interest to declare.

FUNDING INFORMATION: This work was supported by AllerGEN NCE; Canadian Institute of Health Research (CIHR); the Montreal Children's Hospital Foundation (MCHF); and the McGill University Health Centre Foundation (MUHCF).

Casey G. Cohen ${ }^{\mathrm{a}}$, Wei Zhao ${ }^{\mathrm{a}}$, Liane Beaudette ${ }^{\mathrm{b}}$, Duncan Lejtenyi ${ }^{\mathrm{b}}$, Bertrand J. Jean-Claude ${ }^{\mathrm{a}}$, Bruce D. Mazer $^{\mathrm{a}, \mathrm{b}}$

${ }^{a}$ Research Institute of the McGill University Health Centre (RI-MUHC), Montreal, QC, Canada.

${ }^{b}$ Division of Allergy and Immunology, Department of Pediatrics, Montreal Children's Hospital, Montreal, $Q C$, Canada

Correspondence:

Dr. Bruce D. Mazer, Division of Allergy and Immunology, Department of Pediatrics, Montreal Children's Hospital, H4A 3J1, Montreal, QC, Canada. 
Email: bruce.mazer@mcgill.ca

Supporting Information

Additional supporting information may be found online in the Supporting Information section.

Supporting Information:

\section{METHODS}

\section{Physical Peanut Processing}

Commercially available peanuts (Montreal Food Store, Canada) were purchased raw and shelled. Peanuts were roasted in a convection oven at $150^{\circ} \mathrm{C}$ for 30 minutes or were autoclaved in a tabletop autoclave at $136^{\circ} \mathrm{C}(2.5 \mathrm{~atm})$ for 30 minutes. Additionally, roasted peanuts were autoclaved (Roast-Auto) and autoclaved peanuts were roasted (Auto-Roast). Analyses were performed in comparison with raw peanut (unprocessed).

\section{Defatting into flour}

Raw, roasted and autoclaved peanuts (6 to 12 of each) were ground into a smooth paste using a coffee grinder (Hamilton Beach/Proctor-Silex, ON, Canada). The paste was then suspended in hexanes and the peanut flour was collected by filtration under vacuum.

\section{Preparation of Protein Extracts}

Dry peanut flours were processed into whole protein extracts using a $20 \mathrm{mM}$ Tris Buffer (pH 8.5), following the protocol optimized by Walczyk et al.26 Extract concentrations were determined by Bradford Assay27 using bovine serum albumin (BSA, Sigma-Aldrich) as a standard. Concentrations were adjusted to equal values across processing conditions accordingly.

\section{Western Blot Analysis}

Whole protein extracts were diluted to concentrations of $1 \mathrm{mg} / \mathrm{mL}$ and separated by SDS-PAGE under reducing conditions $(2.5 \% \beta$-mercaptoethanol). Membranes were incubated with rabbit anti-Ara h 1 , Ara h 2 or Ara h 8 polyclonal antibody $\left(1: 1,000,4^{\circ} \mathrm{C}\right.$ overnight; Indoor Biotechnologies, VA, USA). Bound antibodies were visualized using horseradish peroxidase (HRP)-conjugated donkey anti-rabbit IgG monoclonal antibody (1:1,000, 1h room temperature; BioLegend, CA, USA) and Clarity/Clarity Max enhanced chemiluminescence (ECL) substrates (Bio-Rad Laboratories, Canada). Products were visualized with the ChemiDoc XRS+ Imaging System (Bio-Rad Laboratories).

\section{Relative Quantification of Allergens}

Relative levels of Ara h 1, Ara h 2 and Ara h 8 in peanut extracts were quantified using the enzyme-linked immunosorbent assay (ELISA). Polystyrene plates (96-well) were coated overnight at $4^{\circ} \mathrm{C}$ with the different protein extracts at a range of concentrations (maximum $1 \mu \mathrm{g} / \mathrm{mL}$ for Ara $\mathrm{h} 1$ and Ara $\mathrm{h} 2$, maximum 1 $\mathrm{mg} / \mathrm{mL}$ for Ara h 8). Following blocking with $1 \%$ BSA, rabbit anti-Ara h 1, Ara h 2 or Ara h 8 polyclonal antibody (1:1000, $50 \mu \mathrm{L} /$ well, $2 \mathrm{~h}$ room temperature; Indoor Biotechnologies) was used as the primary antibody and HRP-conjugated donkey anti-rabbit IgG monoclonal antibody $(1: 1,000,50 \mu \mathrm{L} /$ well, $1 \mathrm{~h}$ room temperature; BioLegend) was used for detection. Following incubation with 3,3',5,5'-Tetramethylbenzidine (TMB) substrate (BioLegend), optical density (OD) values were measured at $450 \mathrm{~nm}$ with reference at 570 nm.

\section{Determination of Specific IgE Responses}

The specific IgE-binding capacity of the proteins of the various processing conditions was analyzed using the ELISA protocol described above. The serum of 4 patients highly allergic to peanuts diluted 1:1000 in 1\% BSA were each used as the primary antibody $(50 \mu \mathrm{L} /$ well, $2 \mathrm{~h}$ room temperature). Biotinylated polyclonal goat anti-human IgE antibody (1:20,000, $50 \mu \mathrm{L} /$ well, $1 \mathrm{~h}$ room temperature; Bethyl Laboratories Inc., TX, 
USA) followed by incubation with HRP-streptavidin (1:3,000, $50 \mu \mathrm{L} /$ well, $1 \mathrm{~h}$ room temperature; BioLegend) were used for detection.

A serial dilution of recombinant human IgE antibody at $50 \mathrm{ng} / \mathrm{ml}$ (ELISA Ready-SET-Go! Kit, Thermo Fisher Scientific, ON, Canada) binding to goat anti-human IgE capture antibody (1:1,000; Bethyl Laboratories Inc.) coated to the plate was used to construct a standard curve by plotting known concentrations versus $\mathrm{OD}$ values at $450 \mathrm{~nm}$ with reference at $570 \mathrm{~nm}$.

\section{Skin Prick Testing}

Nine peanut-allergic subjects and three non-allergic healthy controls aged 19 to 29 years old were recruited to the Montreal Children's Hospital for Skin Prick Testing (SPT) after obtaining informed consent. Subjects were considered allergic based on previous history suggestive of immediate allergy to peanut and the presence of previous positive SPT to peanut (wheal diameter greater than $7 \mathrm{~mm}$ ), detection of serum-specific $\operatorname{IgE}$ to peanut proteins $(>0.35 \mathrm{kU} / \mathrm{L})$ and/or a positive oral food challenge to peanut.

Peanuts processed by roasting, autoclaving, roasting then autoclaving, and autoclaving then roasting, along with raw peanuts, were further processed into protein extracts as described above. Extracts of each condition were diluted to equal concentrations equivalent to the commercial standard peanut extract (Allergy Canada Limited, Thornhill, ON, LOT No: 3467710) as measured by Bradford Assay. The SPTs were conducted by placing a drop of each extract, as well as the standard commercial extract, on the forearm and making a small scratch on the arm using a solid bore needle. Saline diluent and Histamine $(1 \mathrm{mg} / \mathrm{mL}$, ALK-Abello Pharm., Inc., ON, Canada) were used as negative and positive controls, respectively. After 10 minutes, the size of the wheal diameter was measured (in $\mathrm{mm}$ ). All skin tests were performed by an experienced allergy nurse. Research Ethics Board (REB) approval was obtained from the McGill University Health Centre (MUHC-REB 2020-5745).

\section{Statistical Analyses}

All results were statistically analyzed using GraphPad Prism Version 5.00 (GraphPad Software, San Diego, CA, USA). Analysis of Variance (ANOVA) was used to determine significant differences between normalized IgE binding values of each processing condition. A two-tailed, paired Student's t-test was used to determine significant differences between wheal diameters using raw and autoclaved peanut protein extracts. The level of significance was set at $\mathrm{p}<0.05$ in both cases. 